\title{
Perfect Homeostasis: pH
}

\section{R. Vinodh Rajkumar.}

Physiotherapist, Medical Anthropologist \& Gerontologist, Director - Prabhanjeet Fitness Research

Institute OPC Private Limited, Bangalore, Karnataka, India.

iD https://orcid.org/0000-0002-6656-3178

\section{ABSTRACT}

Physiotherapists, Dieticians, and Exercise professionals have much better scope to become trailblazers in the forthcoming years to revamp the public health system and set spectacular trends in interdisciplinary health care for the people. Meanwhile, they must scrupulously engage in research pursuits and the dissemination of advanced knowledge in health and disease. A macrocosm of research literature in all the medical super-specializations are currently available but they would go futile if they are not unified and channelized for holistic and equitable health care. Human health is attained or maintained by acquiring homeostasis to coordinate and control a wide range of physiologic parameters within Narrow Homeostatic Range [NHR] that encompasses body temperature, $\mathrm{pH}$, blood glucose level, blood cholesterol level, blood pressure, heart rate, respiratory rate, etc., Homeostasis tightly regulates NHR of all the physiologic parameters to develop homeostatic competence. Homeostatic competence is quite impossible without all the physiologic parameters harmoniously integrating with each other and working in their respective NHR. Physiologic parameters in NHR can be called as homeostatic factors. Deviation of even one physiologic parameter from its NHR could lead to temporary or permanent health disturbance due to disrupted homeostasis. But diseases tend to arise as a result of disturbance in all the physiologic parameters at the same time, though some or many of them could go unnoticed in the contemporary diagnostic procedures or due to lack of appropriate diagnosis. The homeostatic competence [or perfect homeostasis] of individuals that resists non-communicable diseases could also help resisting infectious diseases or vice versa. If only the data of physiologic parameters responsible for preventing non-communicable diseases are available, the health care professionals should be able to get a standpoint about the immunologic fitness of an individual [immunocompetent or immunocompromised] or if only the data of physiologic parameters responsible for immunologic fitness are available, the health care professionals should be able to get a standpoint about an individual's ability to resist non-communicable diseases. These postulates on homeostatic competence can be made irrefutable by carefully reviewing the literature to establish associations between various homeostatic factors and the versatile roles played by each homeostatic factor in [i] preventing infectious diseases and non-communicable diseases [ii] maintaining $\mathrm{pH}$ and [iii] ensuring perfect homeostasis. Advancing Estimation and Gradation of Immunologic Status [AEGIS] can also be constructed putting together all homeostatic factors. Under the beneficial ecological conditions and lifestyle, human body as a biochemical machine would strive to adapt and converge all the versatile efforts of homeostatic factors to create perfect homeostasis for itself in association with a NHR for $\mathrm{pH}$. Undoubtedly, at any age, Homeostatic Excellence Actuates Life Through Health [HEALTH] is naturally possible.

KEY WORDS: Homeostasis, pH, Acid-base balance, Exercise, Nutrition, Ageing, Immunity, Infections, Non-communicable diseases, Public health

Address for correspondence: R.Vinodh Rajkumar, Physiotherapist, Medical Anthropologist \& Gerontologist, Director - Prabhanjeet Fitness Research Institute OPC Private Limited, Bangalore, Karnataka, India. E-Mail: dreamofkalam@rediffmail.com

Access this Article online

Quick Response code

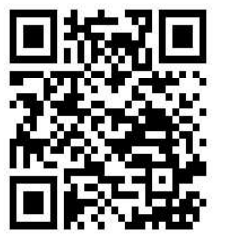

DOI: 10.16965/ijpr.2021.213
Journal Information

International Journal of Physiotherapy and Research

ISSN (E) 2321-1822 | ISSN (P) 2321-8975 https://www.ijmhr.org/ijpr.html

DOI-Prefix: https://dx.doi.org/10.16965/ijpr

(cc) $\mathrm{ET}-\mathrm{N} \mathrm{N}-\mathrm{SH}$

Article Information

Received: 30 Nov 2021

Peer Review: 01 Dec 2021

Revised: None
Accepted: 22 Jan 2022

Published (O): 11 Feb 2022

Published (P): 11 Feb 2022 


\section{INTRODUCTION}

Human health is attained or maintained by acquiring homeostasis to coordinate and control a wide range of physiologic parameters within Narrow Homeostatic Range [NHR] that encompasses body temperature, $\mathrm{pH}$, blood glucose level, blood cholesterol level, blood pressure, heart rate, respiratory rate, etc., Homeostasis tightly regulates NHR of all the physiologic parameters to develop homeostatic competence. Homeostatic competence is quite impossible without all the physiologic parameters harmoniously integrate with each other and work in their respective NHR. Physiologic parameters in NHR can be called as homeostatic factors. Deviation of even one physiologic parameter from its NHR could lead to temporary or permanent health disturbance due to disrupted homeostasis. But diseases tend to arise as a result of disturbance in all the physiologic parameters at the same time, though some or many of them could go unnoticed in the contemporary diagnostic procedures or due to lack of appropriate diagnosis. Plasticity of the body could still enable the individuals to live long despite overwhelming co-morbidities and unhealthy habits but how the body of these individuals manage all the external challenges [like gravitational force, climate] remains mysterious. "Homeostasis is not static and unvarying; it is a dynamic process that can change internal conditions as required to survive external challenges. The health and vitality of the organism can be said to be the end result of homeostatic regulation. Disruption of homeostatic mechanisms is what leads to disease, and effective therapy must be directed toward re-establishing these homeostatic conditions" [1]. "The body's many functions, beginning at the cellular level, operate as to not deviate from a narrow range of internal balance, a state known as dynamic equilibrium, despite changes in the external environment. Those changes in the external environment alter the composition of the extracellular fluid surrounding the individual cells of the body, but a narrow range must be maintained to stave off the death of cells, tissues, and organs" [2]. Human life requires a tightly controlled $\mathrm{pH}$ level in the serum of about 7.4, a slightly alkaline range of 7.35 to 7.45 , to survive [3]. "Multicellular organisms depend on two mechanisms to survive: the regulation and ability to store energy to prevent starvation and the ability to fight against infection. Synergic interactions between metabolism and immunity affect many systems underpinning human health" [4]. The homeostatic competence [or perfect homeostasis] of individuals that resists non-communicable diseases could also help resisting infectious diseases or vice versa. If only the data of homeostatic factors responsible for preventing non-communicable diseases are available, the health care professionals should be able to get a standpoint about the immunologic fitness of an individual [immunocompetent or immunocompromised] or if only the data of homeostatic factors responsible for immunologic fitness are available, the health care professionals should be able to get a standpoint about an individual's ability to resist non-communicable diseases. These postulates on homeostatic competence can be made irrefutable by carefully reviewing the literature to establish associations between various homeostatic factors and the versatile roles played by each homeostatic factor in [i] preventing infectious diseases and non-communicable diseases [ii] maintaining $\mathrm{pH}$ and [iii] ensuring perfect homeostasis.

\section{Homeostatic Competence [or Perfect Homeo- stasis]}

Under the beneficial ecological conditions and lifestyle, human body as a biochemical machine would strive to adapt and converge all the versatile efforts of homeostatic factors [cells, organs, specialized functions] to create perfect homeostasis for itself in association with a NHR for $\mathrm{pH}$. Factors of homeostatic competence should be precisely measurable and their versatile associations with health or diseases or both should have been experimentally proven, and meet the standards of logical reasoning also.

a. Red Blood Cells and Hemoglobin: "The paramount function of the red blood cells [RBCs] is generally reckoned to be oxygen carriage but 
the RBCs are both mechanical and biochemical barriers against infections, bacteria, and blood parasites [5]. Anemia is relatively common in patients with diabetes mellitus, and low hemoglobin concentration contributes to many clinical aspects of diabetes mellitus or its progression [6]. Low hemoglobin concentration in patients with diabetes mellitus is associated with a more rapid decline in glomerular filtration rate than that of other kidney diseases [7]. " Oxyhemoglobin is a stronger acid than deoxyhemoglobin. Oxygenation of hemoglobin causes an increase in net titratable hydrogen ion due to the Haldane effect. As the oxygen saturation of hemoglobin [sO2] increases, the base excess is changed in the acidic direction, or as the $\mathrm{SO}_{2}$ decreases, the base excess is changed in alkaline direction. The changes in the level of the enzyme carbonic anhydrase in RBC are related to the changes in $\mathrm{pH}, \mathrm{pCO} 2$, and bicarbonate levels in the blood" [8].

b. White Blood Cells [WBC]: White blood cells play a most important role in phagocytosis and immunity and therefore in defense against infection [9]. An elevated WBC count [even in the normal range] is closely related to various components of metabolic syndrome [body mass index, hypertension, and triglyceride level] but not related to insulin resistance in type 2 diabetes [10]. An elevated leukocyte count even within the normal range was associated with chronic complications in type 2 diabetes and can be used to predict development of micro and macro vascular complications in patients diagnosed with type 2 diabetes [11]. "In general, the clinical acidaemias are accompanied by immunodeficiency, including a decrease in white cell numbers, $\gamma$ globulins, and mitogenic response and in many cases, the immunodeficiency is reversed on correction of the acidosis" [12].

c. Temperature: "Fever can be deadly, but in moderation it could have some surprising upsides. Bacteria and viruses find it easier to replicate and infect cells at temperatures below $37^{\circ} \mathrm{C}$. By increasing your body temperature, you may be slowing down the ability of a virus to multiply, says Davis. It also seems that the immune system works more efficiently when the body gets hotter. Immune cells that act as first responders to infection, such as dendritic cells, macrophages and neutrophils, have been shown to arrive at the scene faster, and have an improved capacity to engulf and destroy infectious agents at $38^{\circ} \mathrm{C}$ to $40^{\circ} \mathrm{C}$. Permitting a fever in the viral condition is likely to allow your immune system to do its job - as it has been designed by millions of years of evolution - better, says Peters" [13]. Febrile patients with bacteraemia develop a number of acid-base and electrolyte disturbances attributed to various pathogenetic mechanisms which play a significant role in the morbidity and mortality of these patients [14]. "A crucial determinant for the generation of adaptive immunity is the high rate of lymphocyte trafficking through lymphoid organs. Once lymphocytes gain entry into lymphoid organs, there is evidence that their ability to respond to stimulatory signals is also enhanced by febrile temperatures" [15]. Type 1 and 2 diabetes mellitus are both associated with reduced ability to maintain core temperature during thermal stress [16]. "Recurrent dehydration in people regularly exposed to high temperatures seems to be resulting in an unrecognised cause of proteinuric chronic kidney disease. However, beyond heat waves and extreme temperatures, there is a seasonal variation in glomerular filtration rate that may contribute to the onset of renal failure and electrolyte disorders during extremely hot periods" [17].

d. Blood Pressure [BP]: "Hypertension is also accompanied by infiltration of the adventitia and perivascular adipose tissue by inflammatory immune cells including macrophages." [18]. "Hypertension is related to impaired metabolic homeostasis and can be regarded as a metabolic disorder. A high-salt/fat diet may disrupt the gut barrier, which results in systemic inflammation, insulin resistance, and increased BP" [19].

"Hyperinsulinemia caused by insulin resistance stimulates sodium reabsorption, enhances sodium retention, and increases circulating plasma volume. Hyperinsulinemia also stimulates both the renin-angiotensin-aldosterone system [RAAS] and the sympathetic nervous 
system, resulting in the acceleration of atherosclerosis through the hypertrophy of vascular smooth muscle cells, which contributes to increased peripheral vascular resistance" [20].

e. Heart rate [HR] or Resting Heart Rate [RHR]: "Increased physical fitness is associated with a decreased HR at rest, improved autonomic function, and lower risk of cardiovascular events. A higher HR may itself directly induce an inflammatory response through an increased frequency of mechanical stress on the vascular endothelium" [21]. Female gender, lower hemoglobin, obesity, and more $\mathrm{BMI}$, more blood glucose levels, more LDL, and more systolic blood pressure are associated with more RHR [22]. "Increased heart rate and reduced heart-rate variability are associated with an autonomic imbalance in favour of the sympathetic system to play a more important role in the process of atherosclerosis" [23].

Individuals with diabetes may not only be at increased risk for poorer outcomes because of their diabetes but perhaps also because of their significantly higher RHRs [24]. "During the 6-day viral infection period, HR increased significantly by an average of $11 \mathrm{bpm}$ in the supine position and by $22 \mathrm{bpm}$ in the standing position. Orthostatic test is likely to be useful to detect viral diseases early on when implemented in daily routine" [25].

f. Breathing: Breathing rate/rhythm reveals overall health of an individual and numerous health benefits of impeccable breathing/ breathing exercises are 'Pulmonary panacea' [26]. "Autonomically optimised respiration" would appear to be in the band of 6-10 breaths per minute, with an increased tidal volume that is achieved by diaphragmatic activation and this respiratory modulation of autonomic flow is capable of achieving optimal sympathovagal balance" [27]. "Innate critical defensive abilities of lungs are usually able to maintain near-sterility of the lungs without the intervention of 'conventional' cells of the immune system" [28]. Breathing-induced oscillations in $\mathrm{CO}_{2}$ abundance produced large shifts in pH toward levels of alkalinity that are antimicrobial, which may make important contributions toward innate immunity in the lung [29]. "Lung and kidney function are intimately related in both health and disease. Respiratory changes help to mitigate the systemic effects of renal acid-base disturbances" [30]. "Hypoxia and immunity are highly intertwined at clinical, cellular, and molecular levels. The optimal Pao2 and oxygen delivery in critically ill patients are likely to depend on diagnosis and comorbidities, and clinicians should be aware that their oxygen therapy may affect not only saturation but also the inflammatory host response. As clinical guidelines on optimal oxygenation are currently not present, ongoing clinical trials exploring the feasibility of liberal versus restrictive oxygenation are highly warranted and currently in progress; these could further pave the way toward individualized oxygenation therapy" [31]. Since respiratory failure in Diabetic Ketoacidosis [DKA] is associated with increased morbidity and mortality, the recognition and treatment of several electrolyte and metabolic derangements have the potential to improve outcomes in DKA [32]. Breathing training was effective in improvement of physical function, and psychological problems in patients with chronic stroke [33].

g. Cholesterol: Unbalanced lipid metabolism contributes to the development of a wide range of immune disorders including autoimmune diseases, allergies, cancers, and infectious diseases [34]. Dyslipidemia is common in diabetes and there is strong evidence that cholesterol lowering improves cardiovascular outcomes, even in patients with apparently unremarkable lipid profiles [35]. Low HDL cholesterol is a strong risk factor for both atherothrombotic and nonatherothrombotic ischemic stroke [36]. In most cases of essential dyslipidaemia and in certain cases of secondary dyslipidaemia, of paradoxical aciduria, namely aciduria of much greater extent was observed than might have been expected on the basis of plasma $\mathrm{pH}$ value [37].

h. Blood glucose: Blood glucose concentration is under tight metabolic control and is maintained within normal limits by glycogenolysis, gluconeogenesis, glycolysis and insulin/glucagon secretion [38].

"Both low and high glycemic conditions can 
modulate immune function. States of undernourishment depress the immune system, and in the same way, excessive intake of nutrients, such as an obesity state, compromise its functioning" [4]. A better understanding of how immune dysfunctions occur during hyperglycemia can lead to novel treatments to improve the outcome of infectious disease treatment in Type 2 Diabetes Mellitus patients [39]. The diabetics may suffer from acid-base and electrolyte disorders due to complications of diabetes mellitus and the medication they receive [40]. $1 \mathrm{mmol} / \mathrm{l}$ lower usual fasting glucose level was associated with lower risk of stroke and cardiovascular disease [41].

i. Hormonal interplay: Infections and stress, immune responses, and hormones are interconnected, ensuring immune competence to deal with immediate threat of overwhelming infection and metabolic collapse [42]. Growth hormone $[\mathrm{GH}]$ and insulin-like growth factor 1 [IGF-1], especially the former, have immunoregulatory effects in addition to anabolic effects [43]. Thyroid dysfunctions and the metabolic syndrome are the two most common endocrine disorders with a substantial overlap [44]. In humans, glucocorticoid deficiency is commonly associated with a defective immune response and recurrent infections [45]. "Glucocorticoids are widely used as potent anti-inflammatory and immunosuppressive drugs to treat a wide range of diseases. Glucocorticoid-induced diabetes mellitus [GIDM] is a common and potentially harmful problem in clinical practice, but is often difficult to detect in clinical settings" [46].

Clinical states of hyperglucocorticoidism are associated with renal metabolic alkalosis [47]. Significant involvement of the immune system, especially adaptive immunity, was found in the genesis of hypertension and organ damage induced by primary aldosteronism [48]. Aldosterone's most important physiological functions concern maintenance of sodium balance, potassium homeostasis, and excretion of hydrogen ions [49]. Excess deposition of adipose tissue and excess aldosterone production are closely associated [50]. It is well studied that females generate more robust and potentially protective humoral and cell-mediated immune responses following antigenic challenge than their male counterparts [51]. "In young women, estradiol and progesterone primarily control reproduction, but they also affect fluid regulation. Estradiol lowers the operating point for osmoregulation of arginine vasopressin and thirst and increases plasma volume" [52].

j. Liver: The human liver is usually perceived as a non-immunological organ engaged primarily in metabolic, nutrient storage and detoxification activities but hepatocytes perform a number of important immunological roles too [53]. The liver is a key, frontline immune tissue positioned to detect, capture and clear pathogens entering the body via the gut [54]. The liver enzymes Alanine Transaminase [ALT] and Aspartate Transaminase [AST], Alkaline Phosphatase [ALP] and Gammaglutamyl Transferase [GGT] are raised in liver disease but some of these enzymes are also present in other tissues and consequently may be raised in other conditions [55]. Although mild elevations in liver enzymes are associated with features of the metabolic syndrome, only raised GGT is an independent predictor of deterioration of glucose tolerance to Impaired Glucose Tolerance or diabetes [56]. "Bilirubin can neutralize free radicals and prevent peroxidation of lipids. In addition, there is evidence that it protects the cardiovascular system, neuronal systems, the hepatobiliary system, the pulmonary system and the immune system" [57]. Patients with liver diseases may have multiple co-existing metabolic acid-base abnormalities [58].

k. Kidneys: Kidney failure increases susceptibility to infection and promotes exaggerated inflammatory responses, both of which increase mortality, due to disruption in its central role in electrolyte homeostasis and the removal of toxins [59]. In health, the kidneys contribute to immune homeostasis, while components of the immune system mediate many acute forms of renal disease and play a central role in progression of chronic kidney disease [60]. The Kidney is a main target of organ damage in hypertension, and long-term exposure to elevations in blood pressure [BP], 
even within the normotensive range, can induce early renal damage [61]. Determination of serum creatinine concentration is recommended in all patients with hypertension as a marker of target organ damage [62].

In uremia, both defective renal metabolic activities and impaired glomerular filtration resulting in the accumulation of uremic toxins interfere with the immune system associated with increased risk for cardiovascular events [63].

Uric acid is a strong peroxynitrite scavenger and antioxidant and, hypertension is also positively associated with high serum uric acid levels [64]. Kidneys have two significant roles in maintaining acid-base balance; namely, they reabsorb bicarbonate from urine and excrete hydrogen ions in the form of ammonium into urine [65].

I. Platelets: "Platelets are underappreciated orchestrator of immune system [66]. In addition to their role in coagulation and healing, platelets also act as the immune system's first responders when a virus, bacterium, or allergen enters the bloodstream [67]. Essential hypertension is associated with increased risk of arterial thrombotic disease and enhanced platelet activity contributes significantly to this phenomenon [68]. Change of the $\mathrm{pH}$ from acidosis to 7.0 and even to slight alkalosis induces platelet aggregation, platelet calcium and serotonin release, as well as platelet factor II availability [69]. Platelet activation plays a key role in atherothrombosis in type 2 diabetes mellitus [T2DM] even in the earlier stages of disease and in the preclinical phases [70].

Elevated platelet counts can also occur, usually in association with diseases in the elderly, and can result in either excess clotting or even abnormal bleeding [71].

Platelet indices like platelet distribution width [PDW], mean platelet volume [MPV], plateletcrit [PCT], and blood platelet count [PLT] serve as early diagnostic biomarkers and especially MPV and PDW are the simple platelet indices, which increase during platelet activation [72].

m. Plasma proteins: The human plasma comes into contact with virtually all the cells in the human body and changes to specific, or combinations of, plasma protein biomarkers in relation to disease can be used in screening and diagnosis or in the monitoring of treatment [73]. Total serum protein content provides some information regarding a patient's general status; more clinically useful data are obtained from fractionating the total protein [74]. Normal plasma proteins are weak non-volatile acids but although their concentration is not regulated as part of acid-base homeostasis, hypoproteinemia and hyperalbuminemia per se produce alkalosis and acidosis, respectively [75].

"Plasma proteins are the most abundant substances in the plasma and are present in three major types, namely, albumin, globulins, and fibrinogen. Albumin helps maintain the colloid osmotic pressure of the blood that helps to maintain a balance between the water inside the blood and that in the tissue fluid, around the cells" [76]. Immunoglobulins constitute about $20 \%$ of the protein in plasma and being the major antibody in secretions, IgA is found in saliva, tears, colostrum, intestinal, genital tract and respiratory secretions [77]. Low serum AG ratio is an independent predictor for Chronic Kidney Disease [CKD] development and exhibits a stronger predictive value than other inflammatory markers and these findings suggest that determining serum AG ratio may be more valuable for predicting adverse kidney outcomes in non-CKD populations [78].

Elevated Inflammation-sensitive plasma proteins [ISP] predict a large weight gain in middle-aged men and this relation could contribute to the relation between inflammation, the metabolic syndrome, and cardiovascular disease [79]. In nephron-loss disease, both excretion and endogenous catabolism of low molecular weight proteins are diminished, thus, these proteins accumulate in the blood [80].

\section{Advancing Estimation and Gradation of Immu-} nologic Status [AEGIS]: Each factor of homeostatic competence is capable of resisting diseases [infectious and non-communicable diseases] and maintains an association with 
acid-base balance. For instance, as discussed earlier, functions of white blood cells are linked to immunity, $\mathrm{pH}$ and metabolic homeostasis, therefore if the white blood cells are in the NHR, an individual should be regarded competent to resist infections and metabolic disorders because the body's $\mathrm{pH}$ is also perfect. If WBCs are functioning optimally, it is very likely that other factors of homeostatic competence are also functioning optimally in their NHR to ensure homeostatic competence. Medical professionals should try to find answers to these questions; [i] Are there individuals in the world with all the physiologic parameters in the best NHR? [ii] Has any inter-disciplinary team of medical professionals come across individuals with all the physiologic parameters in the best NHR? [iii] Is it possible to rectify the altered/disturbed NHR of any physiologic parameters of the patients only through medicines [without optimal nutrition, exercise and active breathing]? [iv] Individuals with best NHR of all physiologic parameters would be obviously asymptomatic to both infectious and non-communicable diseases. They remain asymptomatic to diseases because they possess quintessential resistance to morbidities. Why they should also be viewed as a possible source of transmission of infectious diseases? [v] How do medicines and vaccines favourably act on the body of the individuals with altered/ disturbed NHR of various physiologic parameters due to multi-morbidities? [vi] Are there no evidences for altered/disturbed NHR of individuals that got rectified only by food, exercise and lifestyle modifications without intake of medicines? [vi] Why do the individuals with best NHR of all the physiologic parameters need medicines or vaccines as a misleading or compulsory strategy of health or public health? [vii] A homeostatic factor responsible for defending against non-communicable disease will be defending against infectious disease also or vice versa. Is it not? [viii] The status of immunocompetent or immunocompromise of individuals should be confirmed on the basis of antibody testing [especially before and after vaccinations]. Is it not? [ix] If antibody testing facility is not available or in the absence of proof of antibodies against any infectious disease, various other homeostatic factors [especially WBC count] will suffice to indirectly understand an individual's immunologic status. Is it not? [x] In the best homeostatic state [where NHR of all the physiologic parameters are in the best conditions], pathogens may not be able to cause any destruction, so there is no need for antibody production. During antibody testing for such highly healthy individuals, if there are no antibodies found for a particular infectious disease, will it be misinterpreted as if they are not immune to a particular infectious disease?

An immunocompetent individual would naturally have quintessential NHRs to resist non-communicable diseases also. Ideally, a broad range of quantitative diagnosis must be conducted to know about the health or disease status of an individual based on their immunity, $\mathrm{pH}$ and various other key homeostatic factors explored so far in the medical field. A macrocosm of research literatures in all the medical super-specializations are currently available but they would go futile if they are not unified and channelized for holistic and equitable health care. Any homeostatic factor that is neither quantifiable nor absolutely prove worthy should be better discarded to avoid causing confusions in the health care, and irreparable tragedies in human life due to flawed public health manoeuvres. Advancing Estimation and Gradation of Immunologic Status [AEGIS] for any specific infectious disease can also be objectively constructed by combining all the homeostatic factors including antibody levels [Table - 1]. Grade 1 would indicate that an individual possesses strong immune system and all the homeostatic factors are in NHR, and the whole body is in Perfect Homeostasis. If there are other ways to examine the immunologic status excluding the antibody assay, Grade 1 individuals will still exhibit their robust immune system whilst Grade 6, their immunocompromised condition.

Cells of the innate immune system serve as the first line of defense against pathogens that include monocytes, macrophages, neutrophils, dendritic cells [DCs], natural killer [NK] cells, eosinophils, and basophils [81]. 
Table 1: AEGIS for any specific infectious disease (ranging from "Grade 1: Immunocompetent" to "Grade 6: Severely immunocompromised").

\begin{tabular}{|c|c|}
\hline 1 & $\begin{array}{l}\text { Asymptomatic. No antibodies formed because the pathogen could } \\
\text { not harm the individual. }\end{array}$ \\
\hline 2 & Asymptomatic. Antibodies formed naturally and adequately. \\
\hline 3 & Mild illness. Antibodies formed naturally and adequately. \\
\hline 4 & Severe illness. Inadequate antibodies formed naturally. \\
\hline 5 & Severe illness. Inadequate antibodies formed despite vaccination. \\
\hline 6 & $\begin{array}{l}\text { Severe illness. No antibodies formed despite multiple doses of } \\
\text { vaccinations. }\end{array}$ \\
\hline
\end{tabular}

It is a fundamental property of immunity that no part of our body is cut off from its surveillance, for this reason, although the immune system may seem a less substantial thing than an organ such as the heart or the liver, in aggregate, immunity consumes enormous resources, producing the large number of cells that it depends on for successful functioning [82]. In humans, sexual dimorphism in the immune response has been well demonstrated, with females exhibiting lower infection rates than males for a variety of bacterial, viral, and parasitic pathogens [83]. Systemic acid-base homeostasis is maintained and controlled by the concerted action of various organs including lungs, kidneys, liver, bone, skeletal muscle, and intestine [84]. "Homeostatic control of plasma pH [range 7.38-7.42] - defence of the alkaline environment in the face of massive daily acid production - is an essential requirement for life. This is achieved through three lines of defence: physico-chemical buffering, rapid respiratory changes in $\mathrm{pCO} 2$, and slow renal changes in $\mathrm{H}+$ excretion and $\mathrm{HCO}^{-}$reabsorption and production. Disturbances in acid-base balance are described according to the cause of a primary change in either pCO2 [respiratory acidosis, respiratory alkalosis] or plasma $\mathrm{HCO}^{-}$ concentration [metabolic acidosis, metabolic alkalosis]. Full correction of these acid-base disturbances requires removal of the primary disturbance" [85].

"Master Health Checkup [MHC] is a battery of tests done to detect and identify Non Communicable Diseases [NCDs] early and may help in adopting timely interventions in this era of increasing life style diseases. Biochemical investigations of $\mathrm{MHC}$ include - Haemoglobin, Total WBC count, Total RBC count, Platelets,
ESR, Peripheral Smear, Urine routine [including urine glucose and ketone bodies], Serum electrolytes, Fasting Blood Sugar, Postprandial Blood Sugar, HbA1C, Cholesterol, Triglycerides, HDL, VLDL, Bilirubin [total, indirect and direct], Alkaline Phosphatase [ALP], Aspartate aminotransferase [AST], Alanine aminotransferase [ALT], Gamma-glutamyl transferase [GGT], Albumin, Globulin, Serum Urea, Creatinine, Uric Acid, Thyroid function tests, Prostate Specific Antigen or PAP smear, X-ray, ECG and Abdominal ultrasound" [86].

It is highly unlikely for an individual with many disturbed NHRs as proved by a MHC [failing to meet the benchmark to prevent NCDs] to develop adequate antibodies against any infectious disease. Or if the immunological assessments would prove an individual as Grade 6, it is highly unlikely for such immunocompromised individual to meet the NHR benchmarks to prevent NCDs. Wherever feasible and necessary, it is good to conduct both $\mathrm{MHC}$ and immunological tests together to get complete understanding about an individual. Kinanthropometry should be included in such elaborate diagnosis in which Physiotherapists, Dieticians and Exercise Professionals would play a crucial role.

Narrow Homeostatic Range [NHR]: Even the best health is not a stationary state. Health and homeostatic factors could inevitably fluctuate between the NHRs without permitting diseases. However, health could progress to a higher level along with positive shifts in the NHR of homeostatic factors. NHR is highly individual-specific, hence understanding the health and disease of a person needs his/her previous medical records [quite similar to $\mathrm{MHC}$ ] conducted several times in the lifetime [at least once a year for several years], so that 
personalized health care becomes feasible. We need clinical diagnosis and health care based on our own several years of history about health and disease. Unfortunately, the practice of health care has been relying exclusively on statistical averages or fixed laboratory values [FLV] that apparently belong to healthy people. In addition, in the symptomatic or asymptomatic states, if individuals were found to display the values of physiologic parameters above or below the FLV, attempts are often made to attain health only through medicines. But FLV are derived from healthy people who persistently endeavour to lead healthy lifestyle without relying on medicines.

"Underlying individuals' unique, invaluable, and enigmatic metaphysical qualities, the human organism is, in a physical sense, essentially a self-regulating biochemical machine. At any moment, our thoughts and feelings, our actions, metabolism and physical well-being all stem from the sum of dynamic, intricate biochemistry working within a distinctive genetic context; innumerable biochemical reactions are taking place to prepare the enzymes, hormones, neurotransmitters and all that we need to undertake the tasks required for daily life. We are truly wonderfully crafted. Attention to toxic chemical exposures and environmental health sciences has been expanding at an impressive rate" [87]. "Life on earth depends on appropriate $\mathrm{pH}$ levels in and around living organisms and cells. It would be prudent to consider an alkaline diet to reduce morbidity and mortality of chronic disease that are plaguing our aging population. One of the first considerations in an alkaline diet, which includes more fruits and vegetables, is to know what type of soil they were grown in since this may significantly influence the mineral content" [88]. "Oxygen is critical for multicellular existence. Its reduction to water by the mitochondrial electron transport chain helps supply the metabolic demands of human life. The incompletely reduced, reactive oxygen by-products of this reaction, however, can be quite toxic" [89]. "Succeeding generations of physiologists have added greatly to our knowledge regarding blood. Meanwhile, the phenomena of immunity were discovered. These phenomena have been correlated on the one hand with resistance to infectious diseases and on the other hand with changes in the composition of cellular and extracellular constituents of blood" [90].

Acquiring and preserving NHRs through a healthy lifestyle should be encouraged to enjoy Morbidity-Attenuated Life Years [MALYs]. Sepsis, a life-threatening organ dysfunction, results from a dysregulated host response to invading pathogens that may be characterized by overwhelming systemic inflammation or some sort of immune paralysis [91]. Physical exercise and appropriate diet contribute to $\mathrm{pH}$ homeostasis [92]. The body's core internal temperature has a narrow range and typically ranges 97-99 $\mathrm{F}$ with tight regulation [93]. "Despite the increasing personalization of medicine, surprisingly $\sim 37.0^{\circ} \mathrm{C}\left[98.6^{\circ} \mathrm{F}\right]$ continues as the estimate of normal temperature. Individual differences are simply too great. Personalizing body temperature is needed" [94]. In order to ensure normal body function, the human body is dependent on a tight control of its blood glucose levels by the pancreas within a very narrow range of 4-6 $\mathrm{mm}$ [95]. The cholesterol content of cells fluctuates within narrow limits and reflects synthesis within the cells and the rates of influx and efflux which are related to the metabolism of circulating lipoproteins. Accumulation of cholesterol within arterial smooth muscle cells and fibroblasts is the hallmark of atherosclerosis [96]. Plasma calcium concentration is maintained within a narrow range [8.5-10.5 $\mathrm{mg} / \mathrm{dL}$ by the coordinated action of parathyroid hormone $[\mathrm{PTH}], 1,25[\mathrm{OH}]_{2} \mathrm{D}_{3^{\prime}}$, calcitonin, and ionized calcium [iCa ${ }^{2+}$ itself [97]. The concept of using single criterion of normal blood pressure with systolic blood pressure [SBP] < $140 \mathrm{mmHg}$ and diastolic blood pressure [DBP] $<90 \mathrm{mmHg}$ for all ages is still disputable [98].

The impact of lifestyle should be regularly investigated [at least once a year] using as many biomarkers as possible, and suitable lifestyle adjustments [exercise, food, sleep] should also be made taking into account of the ageing process. C-reactive protein [CRP] is an acute inflammatory protein that 
increases up to 1,000-fold at sites of infection or inflammation [99]. HbA1c is useful in the diagnosis, prevention and monitoring of DM, reflecting the impact of lifestyle and medication on glycaemic control over the past 3 months [100]. The erythrocyte sedimentation rate $[E S R]$ is a common hematology test that may indicate and monitor an increase in inflammatory activity within the body caused by one or more conditions such as autoimmune disease, infections or tumors [101].

Exercise immunology views acute exercise [moderate to vigorous intensity, less than 60 minutes] as an important immune system adjuvant but, in contrast, high exercise training workloads, competition events, and the associated physiological, metabolic, and psychological stress are linked with transient immune perturbations, inflammation, oxidative stress, muscle damage, and increased illness risk [102]. Foods Obstruct and Overcome Diseases - FOOD [103]. Physiological ageing is accompanied by decline in immune system function and immune alteration during ageing increases susceptibility to infections [104]. "Elderly are more prone to suffer from renal insufficiency and/or chronic obstructive pulmonary disease. Furthermore, medication with various drugs, such as diuretics, often affect the acid-base balance in the elderly" [105]. "Figuratively speaking, healthy ageing is a competitive sport. Homeostatic regulatory processes tend to keep the environment of the cells within relatively narrow bounds. A [homeo]dynamic aspect of homeostasis deteriorates with age. Dynamic variations in homeostasis are important for the whole body and for the mitochondria, and probably for maintaining physiological fitness in general" [106]. "Humans can be classified based on the impact of lifestyle on their functional status. Quite a lot of humans in the globe have been 'epigenetically efficient' to resist communicable and non-communicable diseases and enjoy Morbidity-Attenuated Life Years [MALYs] to become an exemplary septuagenarian, octogenarian, nonagenarian and centenarian" [107].

\section{CONCLUSION}

Physiotherapists, Dieticians and Exercise professionals have much better scope to become trailblazers in the forthcoming years to revamp the public health system and set spectacular trends in interdisciplinary health care to the people. In general, medical professionals should incline to learn more about the NHRs of immunocompetent individuals. The protective and resilient functions of the body against infections and non-communicable diseases are not executed by unrelated homeostatic factors. Health is firmly regulated by interconnected homeostatic factors. However, it is possible that these homeostatic factors fail temporarily or permanently to maintain the NHRs and the interconnection between them during morbidities and unhealthy ageing associated with immunosenescence. There is no surprise if the 'epigenetically efficient' healthy individuals remain asymptomatic to infections and non-communicable diseases as they might belong to grade 1 according to AEGIS. The versatile and integrated roles of every component of homeostatic competence are extremely vast and only very little amount of them were briefly explained in this article for recapitulation and ratiocination to establish "Perfect Homeostasis [pH]". Further scientific explorations on the basis of these proposed postulates might reveal that just even one precision diagnosis of any one physiologic parameter would suffice to understand an individual's resistance to diseases, and many other aspects of health like sports performance, fertility and ageing. For instance, the medical professionals cannot blindly say that a patient's hemoglobin level is exclusively low but all other physiologic parameters are in NHR, without comparing the medical assessments about NHRs of the same patient done for several years at regular intervals, at least once a year. A medical intervention can be claimed successful if the patients' physiologic parameters return to their personal NHRs but the contemporary health care usually ignores this fundamental and channelize the precautions and treatments towards an unknown or unrelated physiologic parameter, carrying the risks of irreparable iatrogenic damages or unsuccessful rehabilitation. Such fallacies can even mislead the public health strategies on a 
large-scale. To prove or realize these proposed postulates, it is essential to have at least one documentation of all the physiologic parameters [including kinanthropometry] of every individual during their healthy state in life [not during their diseased state] in order to keep their personal homeostasis as benchmark for complete restoration of health if they acquire morbidities at sometime in future. Homeostatic Excellence Actuates Life Through Health [HEALTH] is possible solely relying on optimal nutrition, physical activity, sleep and spiritual intelligence, at any age.

\section{Conflicts of interest: None}

\section{REFERENCES}

[1]. Billman GE. Homeostasis: The Underappreciated and Far Too Often Ignored Central Organizing Principle of Physiology. Front Physiol. 2020;11:200.

[2]. Libretti S, Puckett Y. Physiology, Homeostasis. (Updated 2021 May 9). In: StatPearls (Internet). Treasure Island (FL): StatPearls Publishing; 2021 Jan.

[3]. Waugh A, Grant A. Anatomy and Physiology in Health and Illness. 10th edition. Philadelphia, Pa, USA: Churchill Livingstone Elsevier; 2007.

[4]. Von Ah Morano, AE, Dorneles, GP, Peres, A, Lira, FS. The role of glucose homeostasis on immune function in response to exercise: The impact of low or higher energetic conditions.J Cell Physiol. 2020; 235: 3169-3188.

[5]. Bishlawy IM. Red blood cells, Hemoglobin and the immune system. Med Hypotheses. 1999 Oct;53(4):345-6.

[6]. Kwon E, Ahn C. Low hemoglobin concentration is associated with several diabetic profiles. Korean J Intern Med. 2012;27(3):273-274.

[7]. Progression of nephropathy in type 2 diabetic patients. Rossing K, Christensen PK, Hovind P, Tarnow L, Rossing P, Parving HH. Kidney Int. 2004 Oct; 66(4):1596-605.

[8]. T. Rajini Samuel (July 18th 2019). Who Is Balancing: Is It RBC or Acid-Base Status?, Erythrocyte, Anil Tombak, IntechOpen, DOI: 10.5772/ intechopen.84768.

[9]. Blumenreich MS. The White Blood Cell and Differential Count. In: Walker HK, Hall WD, Hurst JW, editors. Clinical Methods: The History, Physical, and Laboratory Examinations. 3rd edition. Boston: Butterworths; 1990. Chapter 153.

[10]. Mahdiani A, Kheirandish M, Bonakdaran S. Correlation Between White Blood Cell Count and Insulin Resistance in Type 2 Diabetes. Curr Diabetes Rev. 2019;15(1):62-66.

[11]. M., Naredi; D., Jhavar; D., Krishnan. Study of relationship between WBC count and Diabetic complications. International Journal of Advances in Medicine, 2017;4(4):1128-1132.
[12]. Lardner, A. The effects of extracellular pH on immune function. Journal of Leukocyte Biology, 2001;69:522-530.

[13].Geddes L. The fever paradox. New Sci. 2020;246(3277):39-41.

[14]. Elisaf M, Theodorou J, Pappas H, Siamopoulos KC. Acid-base and electrolyte abnormalities in febrile patients with bacteraemia. Eur J Med. 1993 AugSep;2(7):404-7.

[15]. Evans SS, Repasky EA, Fisher DT. Fever and the thermal regulation of immunity: the immune system feels the heat. Nat Rev Immunol. 2015;15(6):335-349.

[16]. Kenny GP, Sigal RJ, McGinn R. Body temperature regulation in diabetes. Temperature (Austin). 2016;3(1):119-145.

[17]. Alberto de Lorenzo, Fernando Liaño, High temperatures and nephrology: The climate change problem, Nefrología (English Edition), 2017;37(5):492-500.

[18]. Singh MV, Chapleau MW, Harwani SC, Abboud FM. The immune system and hypertension. Immunol Res. 2014;59(1-3):243-253.

[19]. Tanaka M, Itoh H. Hypertension as a Metabolic Disorder and the Novel Role of the Gut. Curr Hypertens Rep. 2019;21(8):63.

[20]. Tanaka M. Improving obesity and blood pressure. Hypertens Res. 2020 Feb;43(2):79-89.

[21]. Whelton SP, Narla V, Blaha MJ, et al. Association between resting heart rate and inflammatory biomarkers (high-sensitivity C-reactive protein, interleukin-6, and fibrinogen) (from the Multi-Ethnic Study of Atherosclerosis). Am J Cardiol. 2014;113(4):644-649.

[22]. Goorakani, Y., Sedigh Rahimabadi, M., Dehghan, A. et al. Correlation of resting heart rate with anthropometric factors and serum biomarkers in a population-based study: Fasa PERSIAN cohort study. BMC Cardiovasc Disord 2020;20:319.

[23]. Ahmad Sajadieh, Olav Wendelboe Nielsen, Verner Rasmussen, Hans Ole Hein, Sadollah Abedini, Jørgen Fischer Hansen, Increased heart rate and reduced heart-rate variability are associated with subclinical inflammation in middle-aged and elderly subjects with no apparent heart disease, European Heart Journal, 2004;25(5): 363-370.

[24]. Peer, N., Lombard, C., Steyn, K. et al. Elevated resting heart rate is associated with several cardiovascular disease risk factors in urban-dwelling black South Africans. Sci Rep 2020;10:4605.

[25]. Hottenrott Laura, Gronwald Thomas, Hottenrott Kuno, Wiewelhove Thimo, Ferrauti Alexander. Utilizing Heart Rate Variability for Coaching Athletes During and After Viral Infection: A Case Report in an Elite Endurance Athlete. Frontiers in Sports and Active Living, 2021;3:205.

[26]. R.Vinodh Rajkumar. Breathing Rate/Rhythm Evaluation Ascertains Total Health (BREATH): The Pulmonary Panacea. Int J Physiother Res 2020; 8(5): 3609 $-3619$.

[27].Marc A. Russo, Danielle M. Santarelli, Dean O'Rourke. The physiological effects of slow breathing in the healthy human. Breathe Dec 2017; 
13(4):298-309.

[28].The lungs at the frontlines of immunity. Nat Immunol 2015;16:17.

[29]. Nael A. McCarty; Breathe-Your immune system is counting on it. J Exp Med 5 April 2021; 218 (4): e20202643.

[30]. Pierson DJ. Respiratory considerations in the patient with renal failure. Respir Care. 2006 Apr;51(4):413-22.

[31]. Harmke D. Kiers, Gert-Jan Scheffer, Johannes G. van der Hoeven, Holger K. Eltzschig, Peter Pickkers, Matthijs Kox; Immunologic Consequences of Hypoxia during Critical IIIness. Anesthesiology 2016; 125:237-249.

[32]. Gallo de Moraes A, Surani S. Effects of diabetic ketoacidosis in the respiratory system. World J Diabetes. 2019;10(1):16-22.

[33]. Lee D. The Effect of Breathing Training on the Physical Function and Psychological Problems in Patients with Chronic Stroke. J Kor Phys Ther 2020;32:146-151.

[34]. Kim D, Chung H, Lee JE, Kim J, Hwang J, Chung Y. Immunologic Aspects of Dyslipidemia: a Critical Regulator of Adaptive Immunity and Immune Disorders. J Lipid Atheroscler. 2021;10(2):184-201.

[35]. Schofield JD, Liu Y, Rao-Balakrishna P, Malik RA, Soran H. Diabetes Dyslipidemia. Diabetes Ther. 2016;7(2):203-219.

[36]. Hackam DG, Hegele RA. Cholesterol Lowering and Prevention of Stroke. Stroke. 2019 Feb;50(2):537541.

[37]. Bernardi P, Descovich GC, Rondina M, Pecoraro F, Lenzi S. Controllo renale dell'equilibrio acido-base in corso di dislipidemie (Renal control of acid-base equilibrium during dyslipidemia). Minerva Med. 1977 Feb 18;68(8):489-508.

[38]. J.C. Murrell, D.J. Mellor, C.B. Johnson, Chapter 27 Anaesthesia and Analgesia in the Foetus and Neonate, Anesthesia and Analgesia in Laboratory Animals (Second Edition), Academic Press, 2008;593608.

[39]. Berbudi A, Rahmadika N, Tjahjadi Al, Ruslami R. Type 2 Diabetes and its Impact on the Immune System. Curr Diabetes Rev. 2020;16(5):442-449.

[40]. Sotirakopoulos N, Kalogiannidou I, Tersi M, Armentzioiou K, Sivridis D, Mavromatidis K. Acidbase and electrolyte disorders in patients with diabetes mellitus. Saudi J Kidney Dis Transpl. 2012 Jan;23(1):58-62.

[41]. Blood Glucose and Risk of Cardiovascular Disease in the Asia Pacific Region. Diabetes Care. Dec 2004, 27 (12) 2836-2842.

[42]. Romesh Khardori, Alys Adamski, Nancy Khardori. Infection, Immunity, and Hormones/Endocrine Interactions, Infectious Disease Clinics of North America, 2007;21(3):601-615.

[43]. Saito H, Inoue T, Fukatsu K, Ming-Tsan L, Inaba T, Fukushima R, Muto T. Growth hormone and the immune response to bacterial infection. Horm Res. 1996;45(1-2):50-4.
[44]. Waring AC, Rodondi N, Harrison S, Kanaya AM, Simonsick EM, Miljkovic I, Satterfield S, Newman $A B$, Bauer DC, Health, Ageing, and Body Composition (Health $A B C$ ) Study. Clin Endocrinol (Oxf). 2012 Jun; 76(6):911-8.

[45]. Kim CJ, Woo YJ, Kim GH, Yoo HW: Familial glucocorticoid deficiency with a point mutation in the ACTH receptor: a case report. J Korean Med Sci 2009;24:979-981.

[46]. Suh S, Park MK. Glucocorticoid-Induced Diabetes Mellitus: An Important but Overlooked Problem. Endocrinol Metab (Seoul). 2017;32(2):180189.

[47]. Hulter HN, Licht JH, Bonner EL Jr, Glynn RD, Sebastian A. Effects of glucocorticoid steroids on renal and systemic acid-base metabolism. Am J Physiol. 1980 Jul;239(1):F30-43.

[48]. Herrada AA, Campino C, Amador CA, Michea LF, Fardella CE, Kalergis AM. Aldosterone as a modulator of immunity: implications in the organ damage. J Hypertens. 2011 Sep;29(9):1684-92.

[49]. Knochel JP, White MG. The Role of Aldosterone in Renal Physiology. Arch Intern Med. 1973;131 (6):876-884.

[50]. Rossi, G., Seccia, T. Changes in aldosterone and obesity-related cardiometabolic risk factors with a 1year weight loss intervention in normotensive overweight and obese young adults. Hypertens Res 2013;36:856-858.

[51]. Aruna Bhatia, Harmandeep Kaur Sekhon, Gurpreet Kaur. Sex Hormones and Immune Dimorphism. The Scientific World Journal, 2014, Article ID 159150.

[52]. Stachenfeld NS. Sex hormone effects on body fluid regulation. Exerc Sport Sci Rev. 2008;36(3):152-159.

[53]. Robinson, M., Harmon, C. \& O’Farrelly, C. Liver immunology and its role in inflammation and homeostasis. Cell Mol Immunol 2016;13:267-276.

[54]. Kubes P, Jenne C. Immune Responses in the Liver. Annu Rev Immunol. 2018 Apr 26;36:247-277.

[55]. Limdi JK, Hyde GM. Evaluation of abnormal liver function tests. Postgraduate Medical Journal 2003;79:307-312.

[56]. Nannipieri M, Gonzales C, Baldi S, Posadas R, Williams K, Haffner SM, Stern MP, Ferrannini E; Mexico City diabetes study. Liver enzymes, the metabolic syndrome, and incident diabetes: the Mexico City diabetes study. Diabetes Care. 2005 Jul;28(7):175762.

[57]. Otero Regino, William, Velasco, Héctor, \& Sandoval, Héctor. The protective role of bilirubin in human beings. Revista colombiana de Gastroenterología, 2009;24(3):293-301.

[58]. Scheiner B, Lindner G, Reiberger T, Schneeweiss B, Trauner M, Zauner C, Funk GC. Acid-base disorders in liver disease. J Hepatol. 2017 Nov;67 (5):10621073.

[59]. Kurts, C., Panzer, U., Anders, HJ. et al. The immune system and kidney disease: basic concepts and clinical implications. Nat Rev Immunol 2013;13:738753. 
[60]. Tecklenborn J, Clayton D, Siebert S, Coley SM. The role of the immune system in kidney disease. Clin Exp Immunol. 2018 May;192(2):142-150.

[61]. Perneger TVNieto FJWhelton PKKlag MJComstock GWSzklo M A prospective study of blood pressure and serum creatinine: results for the "Clue" Study and the ARIC Study. JAMA. 1993;269488-493.

[62]. Schillaci G, Reboldi G, Verdecchia P. High-Normal Serum Creatinine Concentration Is a Predictor of Cardiovascular Risk in Essential Hypertension. Arch Intern Med. 2001;161(6):886-891.

[63]. Cohen G. Immune Dysfunction in Uremia 2020. Toxins (Basel). 2020;12(7):439.

[64]. Ghaemi-Oskouie F, Shi Y. The role of uric acid as an endogenous danger signal in immunity and inflammation. Curr Rheumatol Rep. 2011;13(2):160166.

[65]. Aryal, D. and Jackson, K. Association of Acid-Base Balance in the Renal Proximal Tubule and Blood Pressure Alterations: Potential Role of Local Mediators. Journal of Biosciences and Medicines, 2020;8:26-44.

[66]. Ali RA, Wuescher LM, Worth RG. Platelets: essential components of the immune system. Curr Trends Immunol. 2015;16:65-78.

[67]. Université Laval. (2018, February 1). Unexpected role of platelets in immune response. ScienceDaily. Retrieved November 22, 2021 from www.sciencedaily.com/releases/2018/02/ 180201115733.htm

[68]. Eugenia Gkaliagkousi, Gabriella Passacquale, Stella Douma, Chrysanthos Zamboulis, Albert Ferro, Platelet Activation in Essential Hypertension: Implications for Antiplatelet Treatment, American Journal of Hypertension, 2010;23(3):229-236.

[69]. Chaimoff C, Creter D, Djaldetti M. The effect of $\mathrm{pH}$ on platelet and coagulation factor activities. Am J Surg. 1978 Aug;136(2):257-9.

[70]. Santilli F, Simeone P, Liani R, Davì G. Platelets and diabetes mellitus. Prostaglandins Other Lipid Mediat. 2015 Jul;120:28-39.

[71]. Gregg D, Goldschmidt-Clermont PJ. Cardiology patient page. Platelets and cardiovascular disease. Circulation. 2003 Sep 30;108(13):e88-90.

[72]. Yang K, Tao L, Mahara G, et al. An association of platelet indices with blood pressure in Beijing adults: Applying quadratic inference function for a longitudinal study. Medicine (Baltimore). 2016;95(39):e4964.

[73]. Enroth S. Plasma Proteins and Cancer. Cancers. 2021; 13(5):1062.

[74]. Busher JT. Serum Albumin and Globulin. In: Walker HK, Hall WD, Hurst JW, editors. Clinical Methods: The History, Physical, and Laboratory Examinations. 3rd edition. Boston: Butterworths; 1990. Chapter 101.

[75]. Rossing TH, Maffeo N, Fencl V. Acid-base effects of altering plasma protein concentration in human blood in vitro. J Appl Physiol (1985). 1986 Dec;61(6):2260-5.
[76].https://www.news-medical.net/health/BloodPlasma-Components-and-Function.aspx, dated 23.11.2021

[77].https://www.statpearls.com/ArticleLibrary/ viewarticle /23392\#ref_2186517, dated 23.11.2021

[78]. Park J, Kim HJ, Kim J, Choi YB, Shin YS, et al. Predictive value of serum albumin-to-globulin ratio for incident chronic kidney disease: A 12-year community-based prospective study. PLOS ONE 2020;5(9): e0238421.

[79]. Engström G, Hedblad B, Stavenow L, Lind P, Janzon L, Lindgärde $\mathrm{F}$. Inflammation-sensitive plasma proteins are associated with future weight gain. Diabetes. 2003 Aug;52(8):2097-101.

[80]. Strober W, Waldmann TA. The role of the kidney in the metabolism of plasma proteins. Nephron. 1974;13(1):35-66.

[81]. Chaplin DD. Overview of the immune response. J Allergy Clin Immunol 2010;125(2 Suppl 2):S3-S23.

[82]. Nicholson LB. The immune system. Essays Biochem. 2016;60(3):275-301.

[83]. Shepherd Rebecca, Cheung Ada S., Pang Ken, Saffery Richard, Novakovic Boris. Sexual Dimorphism in Innate Immunity: The Role of Sex Hormones and Epigenetics. Frontiers in Immunology. 2021;11:3559.

[84]. Wagner C, A: Effect of Mineralocorticoids on AcidBase Balance. Nephron Physiol 2014;128:26-34.

[85]. Atherton, John. Acid-base balance: maintenance of plasma $\mathrm{pH}$. Anaesthesia \& Intensive Care Medicine. 2009;10:557-561.

[86]. Sathiyamoorthi S, Anand DP, Muthunarayanan L. Is Master Health Checkup the Answer to Tackle the Rising Non-Communicable Disease Burden in India? - A Cross-Sectional Study. J Lifestyle Med. 2019;9(2):111-118.

[87]. Genuis SJ, Sears ME, Schwalfenberg G, Hope J, Bernhoft R. Clinical detoxification: elimination of persistent toxicants from the human body. ScientificWorldJournal. 2013;2013:238347.

[88]. Schwalfenberg GK. The alkaline diet: is there evidence that an alkaline $\mathrm{pH}$ diet benefits health?. J Environ Public Health. 2012;2012:727630.

[89]. Emin Maltepe, Ola Didrik Saugstad. Oxygen in Health and Disease: Regulation of Oxygen HomeostasisClinical Implications. Pediatr Res 65: 261-268, 2009.

[90]. E. J. Cohn. The Properties and Functions of the Plasma Proteins, with a Consideration of the Methods for their Separation and Purification. Chemical Reviews 194128 (2), 395-417.

[91]. Heming Nicholas, Sivanandamoorthy Sivanthiny, Meng Paris, Bounab Rania, Annane Djillali. Immune Effects of Corticosteroids in Sepsis. Frontiers in Immunology, Vol 9, 2018, 736.

[92]. Aoi W, Marunaka Y. Importance of pH homeostasis in metabolic health and diseases: crucial role of membrane proton transport. Biomed Res Int. 2014;2014:598986.

[93]. Osilla EV, Marsidi JL, Sharma S. Physiology, Temperature Regulation. (Updated 2021 May 7). In: 
StatPearls (Internet). Treasure Island (FL): StatPearls Publishing; 2021 Jan.

[94]. Diamond A, Lye CT, Prasad D, Abbott D. One size does not fit all: Assuming the same normal body temperature for everyone is not justified. PLOS ONE 2021;16(2): e0245257.

[95]. Röder PV, Wu B, Liu Y, Han W. Pancreatic regulation of glucose homeostasis. Exp Mol Med. 2016;48(3):e219.

[96]. Paul John Nestal, Cellular control of cholesterol metabolism. Pathology, 1978;10(2):0031-3025,

[97]. Jeon US. Kidney and calcium homeostasis. Electrolyte Blood Press. 2008;6(2):68-76.

[98]. Lin JD, Chen YL, Wu CZ, et al. Identification of Normal Blood Pressure in Different Age Group (published correction appears in Medicine (Baltimore). 2018 May;97(18):e0685) (published correction appears in Medicine (Baltimore). 2016 Jun 24;95(25):e6777). Medicine (Baltimore). 2016;95(14):e3188.

[99].Sproston Nicola R., Ashworth Jason J. Role of C-Reactive Protein at Sites of Inflammation and Infection. Frontiers in Immunology, 2018;9:754.

[100].Kaiafa G, Veneti S, Polychronopoulos G, et al. Is $\mathrm{HbA1c}$ an ideal biomarker of well-controlled diabetes? Postgraduate Medical Journal 2021;97:380383.
[101].Tishkowski K, Gupta V. Erythrocyte Sedimentation Rate. (Updated 2021 May 9). In: StatPearls (Internet). Treasure Island (FL): StatPearls Publishing; 2021 Jan.

[102].David C. Nieman, Laurel M. Wentz, The compelling link between physical activity and the body's defense system, Journal of Sport and Health Science, 2019;8(3):201-217.

[103].R.Vinodh Rajkumar. Foods Obstruct and Overcome Diseases (FOOD): The Missing Interdisciplinary and Transdisciplinary Public Health Art till Covid-19 Pandemic. Int J Physiother Res 2020;8(5):3586-3594.

[104].Valiathan, R., Ashman, M. and Asthana, D. Effects of Ageing on the Immune System: Infants to Elderly. Scand J Immunol, 2016;83:255-266.

[105]. Nabata T, Morimoto S, Ogihara T. (Abnormalities in acid-base balance in the elderly). Nihon Rinsho. 1992 Sep;50(9):2249-53.

[106].van Beek JH, Kirkwood TB, Bassingthwaighte JB. Understanding the physiology of the ageing individual: computational modelling of changes in metabolism and endurance. Interface Focus. 2016;6(2):20150079.

[107].R.Vinodh Rajkumar. Necessity of an Exclusive Health Care Model for Individuals with Quintessential Epigenetic Efficiency. Int J Physiother Res 2021; 9 (5): 3992-4000.

How to cite this article: R. Vinodh Rajkumar. Perfect Homeostasis: $\mathrm{pH}$. Int J Physiother Res 2022;10(1):4111-4124. DOI: 10.16965/ijpr.2021.213 